\title{
A Method of Computer-aided Modular Fixture Design, Part 1: Creating the Feature-model Repository of Fixture Elements
}

Zhun Wang

Anhui Polytechnic University, China. E-mail: CADCAM_246@126.com

Modular fixture is conventionally designed less concerning the detailed specifications of machine tool. A little of literatures involve the effectively application to the existing CAD systems in CAFD (computer-aided fixture design). The determination of the validity of modular fixture during NC machining lacks for a practical method. This paper put forward that: Firstly, the feature-model repository of elements of modular fixture can be built in CAD packages; the design of modular fixture in NC machining should be accomplished under the concept of NC Manufacturing System (NMS); the Post-NC verification can be applied to check the performance of modular fixture applied in NC machining. Part 1 of the paper focuses on the feature-model repository of the modular-fixture elements. The other jobs will be introduced in the Part 2.

Keywords: Modular Fixture, Feature-model Repository, Typical Part (TP), Serial Part Driven with Table

\section{References}

[1] ALI KEYVANI (2012). Modular Fixture Design for BIW Lines Using Process Simulate, LAP LAMBERT Academic Publishing, ISBN: 978-3848418367.

[2] ALI K. KAMRANI, SA'ED M. SALHIEH (2002). Product Design for Modularity, Springer, ISBN: 9781402070730.

[3] HAlEVI, G., WEILL, R. (2012). Principles of Process Planning: A logical approach, Springer, ISBN: 9789401045445.

[4] JOSHI, P. H. (2013). Jigs and Fixtures, 3 edition, McGraw Hill Education (India) Private Limited, ISBN: 9781259061226.

[5] TANG, S. H., MA, Y. S., CHEN, G. (2004). A Feature-oriented Database Framework for web-based CAx applications. In: Computer-Aided Design \& Applications, Vol. 1, No. 1-4, pp. 117-125.

[6] TAMER M. M. SHAHIN (2008). Feature-Based Design - An Overview, In: Computer-Aided Design \& Applications. Vol. 5, No. 5, pp. 639-653.

[7] WILLEM F. BRONSVOORT, BIDARRA, R., PAULOS, J. (2006). Developments in Feature Modelling. In: Computer-Aided Design \& Applications. Vol. 3, No. 5, pp. 655-664.

[8] FABIAN, M., BOSLAI, R., IŽOL, P., JANEKOVÁ, J., FABIANOVÁ, J., FEDORKO, G., BOŽEK, P. (2015). Use of Parametric 3D Modelling - Tying Parameter Values to Spreadsheets at Designing Molds for Plastic Injection. In: MANUFACTURING TECHNOLOGY. Vol. 15, No. 1, pp.24-31

[9] YUNBO ZHOU, YINGGUANG LI, WEI WANG (2011). A feature-based fixture design methodology for the manufacturing of aircraft structural parts. In: Robotics and Computer-Integrated Manufacturing. Vol. 27, No. 6, pp.986-993.

[10] SOENEN, R., OLling, G. J. (2002). Feature Based Product Life-Cycle Modelling, Springer, ISBN: 9781402073274.

[11] SHIH, R. (2014). Parametric Modeling with Autodesk Inventor 2015, SDC Publications, ISBN: 978-1585038824.

[12] MONKA, P., HLOCH, S., ANDREJ, A., SOMSAK, M., MURGAS, F. (2016). Simulation Tools Used at the Injection Mould Design. In: MANUFACTURING TECHNOLOGY. Vol. 16, No. 3, pp.561-569.

[13] SEDLAK, J., SLANY, M., FIALA, Z., JAROS, A. (2015). Production Method of Implant Prototype of Knee-Joint Femoral Component. In: MANUFACTURING TECHNOLOGY. Vol. 15, No. 2, pp.195-204.

[14] MICHAUD, M. (2012). CATIA Core Tools: Computer Aided Three-Dimensional Interactive Application, 1 edition, McGraw-Hill Education, ISBN: 978-0071700269.

[15] LEE H FELIX, FULTON DAVID (2012). Computer Aided Design with Unigraphics NX7.5, 8 edition, Kendall Hunt Publishing, ISBN: 978-1465200266.

[16] SHAM TICKOO (2012). Creo Parametric 2.0 for Designers, CADCIM Technologies, ISBN: 978-1936646388.

[17] VALENTINO, J. (2010). SolidWorks for Technology and Engineering, 1st edition, Industrial Press, Inc., ISBN: 978-0831134150.

[18] FABIAN, M., STANOVÁ, E., FEDORKO, G., KMEŤ, S., FABIANOVÁ, J., KRAJŇÁK, J. (2016). Parametric CAD Model of a Double-Lay Six Strand Wire Rope. In: MANUFACTURING TECHNOLOGY. Vol. 16, No. 3, pp. 489-496. 